\title{
Reynolds number dependence of scalar fluctuations in a high Schmidt number turbulent jet
}

\author{
Paul L. Miller and Paul E. Dimotakis \\ Graduate Aeronautical Laboratories, California Institute of Technology, Pasadena, California 91125
}

(Received 28 August 1990; accepted 9 January 1991)

\begin{abstract}
The scalar rms fluctuations in a turbulent jet were investigated experimentally, using highresolution, laser-induced fluorescence techniques. The experiments were conducted in a high Schmidt number fluid (water), on the jet centerline, over a jet Reynolds number range of $3000 \leqslant \operatorname{Re} \leqslant 24000$. It was found that the normalized scalar rms fluctuations $c^{\prime} / \bar{c}$ decrease with increasing flow Reynolds number, at least for the range of Reynolds numbers investigated. Since $c^{\prime} / \bar{c}$ is a measure of the inhomogeneity of the scalar field, this implies that high Schmidt number turbulent jets become more homogeneous, or better mixed, with increasing Re. These findings need to be assessed in the context of the documented Reynolds number independence of flame length for Re $>3000$ or 6500 .
\end{abstract}

\section{INTRODUCTION}

The correct description of the probability density function ( $p d f$ ) of the fluctuations of a conserved passive scalar in turbulent flow has been a matter of theoretical and engineering interest for some time. Knowing the pdf of the conserved scalar allows the computation of the amount of chemical product that would be formed in a chemical reaction in which the scalar is transported as a passive quantity. ${ }^{1}$

Experimental evidence and theoretical arguments in the last few years suggest that the turbulent mixing process is sensitive to the value of the molecular diffusion coefficients, even at Reynolds numbers typically regarded as high. We recognize that this remains a controversial proposal, at this writing, especially in the case of turbulent jet mixing. ${ }^{2} \mathrm{We}$ note, in particular, that accepting it would imply that the conserved scalar pdf, as well as other statistics of the mixing process, should be expected to be functions of the flow Reynolds number $\operatorname{Re}=U \delta / \nu$, where $U$ is the local flow velocity, $\delta$ is the local extent of the turbulent flow and $v$ denotes the (molecular) kinematic viscosity, and the Schmidt number $\mathrm{Sc}=v / \mathscr{D}$, where $\mathscr{D}$ is the scalar species (molecular) diffusivity. There is no consensus at this time as to how such effects should be described, let alone predicted, with the associated nonlinear dynamics essentially involving terms in the equations of motion with factors that vanish, multiplied by factors that become unbounded in the limit of $\operatorname{Re} \rightarrow \infty$, or $\mathrm{Sc} \rightarrow \infty$.

Nevertheless, the qualitative dependence on $\mathrm{Sc}$ might be argued on simple grounds, namely, that decreasing the species diffusivity, i.e., increasing the Schmidt number, keeping all other flow and fluid quantities fixed, can only decrease the rate of (molecular) mixing and the amount of mixed fluid. This is corroborated by a comparison of the results of experiments in gas phase shear layers ${ }^{3}(\mathrm{Sc} \sim 1)$ versus those in liquid phase shear layers ${ }^{4}\left(\mathrm{Sc} \sim 10^{3}\right.$ ) at comparable Reynolds numbers.

An a priori argument for the dependence on Reynolds number is not as straightforward. Reliable experimental data at large Reynolds numbers are difficult to obtain. Experiments at high Reynolds numbers must rely on chemically reacting flows to infer the degree of molecular mixing. In the case of gas phase shear layers, the experimental evidence from a single experiment is that the amount of mixing decreases as the Reynolds number increases, albeit slowly. ${ }^{5}$ The evidence shows a Reynolds number dependence in liquid phase shear layers that appears to be weaker, but this conclusion is based on scanter data. ${ }^{4}$

In turbulent jets, the behavior is even less clear. Experimental "flame length" data in liquid jets, ${ }^{6}$ as well as other liquid and gas phase turbulent jet flame length data reviewed in Fig. 1 of Dahm and Dimotakis, ${ }^{7}$ suggest that this quantity may well be independent of both Schmidt number and Reynolds number, at least for Reynolds numbers greater than about $3 \times 10^{3}$, or so, as had been reported in the earlier Weddel1 ${ }^{8}$ (see also Hottel, Ref. 9) findings. The scatter in these data is large enough, however, to have masked a weak dependence on Schmidt number or Reynolds number, not to speak of the likely contamination of the results by buoyancy effects in the case of high heat release, gas phase jet flames. As a case in point, the results of Kristmanson and Danckwerts ${ }^{10}$ suggest that Reynolds number independence of the flame length in a liquid jet does not occur until a Re greater than 6500 , or so.

More recently, direct measurements of the jet fluid concentration pdr in gas phase turbulent jets by Dowling and Dimotakis ${ }^{11}$ suggest a Reynolds number independence, at least in the Reynolds number range from $5.0 \times 10^{3}$ to $4.0 \times 10^{4}$. These authors also compare their estimates of the jet fluid concentration pdf on the jet axis with those measured by other investigators, ${ }^{12-16}$ for Schmidt (Prandtl) numbers ranging from 0.7 to $10^{3}$ and Reynolds numbers ranging from $5 \times 10^{3}$ to $5.2 \times 10^{4}$ (Dowling and Dimotakis, ${ }^{11}$ Fig. 28). While the reported pdf's may not collapse particularly well, it is difficult to assess whether the differences are attributable to Schmidt number or Reynolds number effects, or to experimental difficulties.

Our own investigations of the behavior of the turbulent 
jet fluid concentration fluctuations were undertaken as part of a larger effort to address the role of Reynolds number as a parameter in the dynamics of turbulent jet mixing. The experiments reported here were performed in liquid phase turbulent jets $\left(\mathrm{Sc} \sim 10^{3}\right)$, in a Reynolds number range from $3.0 \times 10^{3}$ to $2.4 \times 10^{4}$. Our measurements permitted direct estimates of the pdf, rms, as well as the spectrum of the conserved scalar fluctuations to be computed.

\section{APPARATUS AND PROCEDURE}

While the experimental setup has been documented previously, ${ }^{6,17}$ the following short description is included for completeness. The experiments were carried out utilizing the facility shown in Fig. 1. A large rectangular water tank, about $1 \mathrm{~m}$ square and $2 \mathrm{~m}$ high, acted as the (discharge) reservoir. Large windows on all sides of the tank provided optical access. To establish the flow, the jet plenum was filled with water tagged by a fluorescent laser dye (sodium fluorescein) and air was sonically metered to drive the jet fluid at constant velocity through a $2.54 \mathrm{~mm}(0.1 \mathrm{in}$.) diam nozzle at the base of the plenum. The beam from an argon- ion laser was passed through appropriate optics and aligned radially through the centerline of the jet. A beam stop prevented reflections from the opposite window. For these measurements, the laser beam optics were designed to generate a small Gaussian waist at the focus. A low laser power of 1.0 $\mathrm{W}$ was used to avoid heating of the dyed fluid in the very small focal volume and to prevent saturation. The plenum dye concentration was kept less than $10^{-6} \mathrm{M}$. Consequently, the much lower concentrations at the measuring station did not significantly attenuate or steer the beam.

A low $f$ \# lens was used to collect light from a short segment centered at the waist of the focused laser beam (on the jet centerline, at $x / d=100$ ) onto a photomultiplier tube, effectively yielding single-point concentration values versus time. An optical low-pass filter eliminated background laser light, transmitting only the frequency-shifted fluorescence. 'A slit spatial filter defined the length of the laser line segment sampled. The slit width was chosen such that the sampling volume was roughly cylindrical, about 80 $\mu \mathrm{m}$ in diameter and less than $50 \mu \mathrm{m}$ in length. The use of the slit rather than a pinhole ensured that any small transverse beam movements did not alter the measurement volume. The long Rayleigh range and latitude in the depth of field

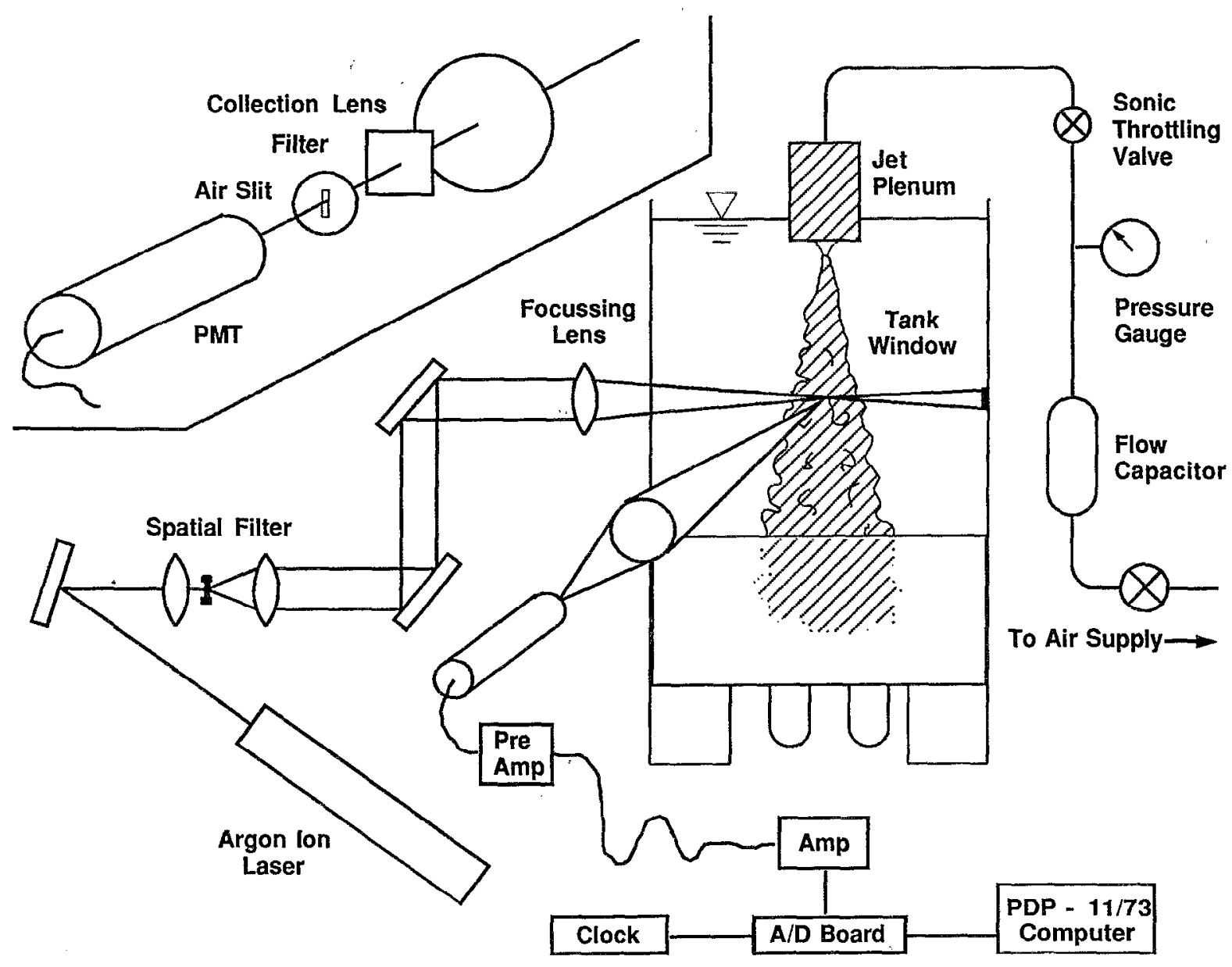

FIG. 1. Experimental apparatus. 
minimized the effect of motion in either of the two horizontal directions. Data acquisition was computer-controlled. The signal amplifier incorporated a three-pole Butterworth filter, with a cutoff frequency set slightly under $10 \mathrm{kHz}$. The data were sampled at $20 \mathrm{kHz}$ for all the runs.

Careful consideration has been given to the reduction of noise in these measurements, especially since we wish to examine the normalized scalar rms fluctuations, $c^{\prime} / \bar{c}$. As described previously, ${ }^{17,18}$ we utilized Wiener filter techniques ${ }^{19,20}$ in processing the data. The issues of buoyancy and attenuation of the laser beam have been examined, and are discussed in Appendix A. We conclude that neither is a factor in these jets. Finally, an important consideration when measuring a quantity such as $c^{\prime} / \bar{c}$ is resolution, since fluctuations at scales smaller than the measuring volume are low-pass filtered. It is therefore necessary to determine that either the smallest scales of the flow are resolved or that the contribution to the fluctuations by scales smaller than the resolution is negligible. We discuss resolution concerns later in this paper.

\section{RESULTS}

For our discussions, the jet Reynolds number is defined as $\operatorname{Re}=U_{0} d / v$, where $U_{0}$ is the jet exit velocity, $d$ is the diameter of the jet nozzle, and $v$ is the kinematic viscosity of the working fluids (water).

Our measurements indicate that the scalar field of a high Schmidt number turbulent jet has, in some sense, not reached an asymptotic state for $R e$ as large as 10000 to 20000 , or more. This is apparent in the behavior of the scalar pdf 's with varying Re. In Fig. 2, we show four sample pdf 's, spanning Reynolds numbers from 3000 to 24000 . One hundred and fifty histogram bins were used to compile each pdf. Approximately 34 large-scale structure passages were captured at the lowest $\mathrm{Re}$, and over 125 at the highest.

These pdf 's display a decreasing relative concentration fluctuation amplitude and imply that the jet is becoming more homogeneous in composition with increasing $\mathrm{Re}$. It could be argued that this may be attributable to worsening resolution, as Rc increases. It turns out, however, that the

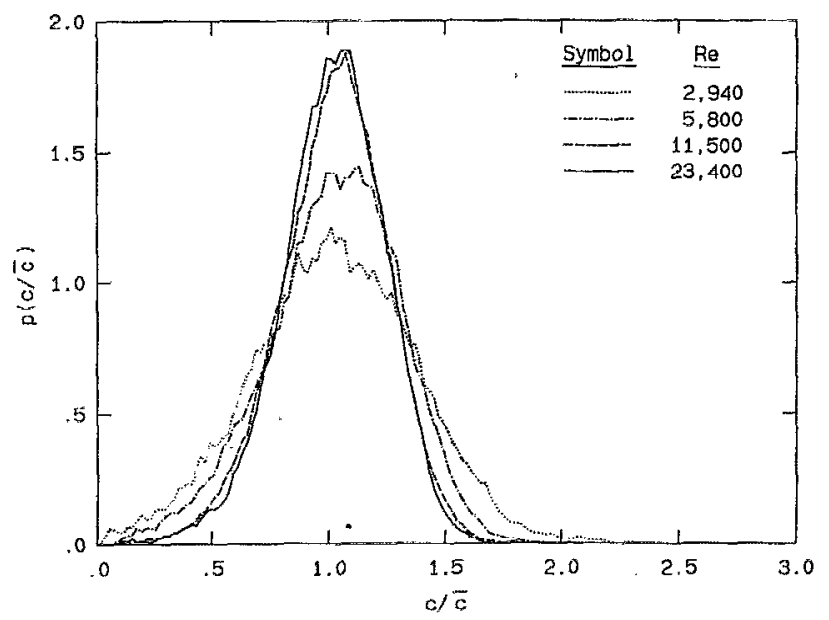

FIG. 2. Scalar pdf's for varying $\operatorname{Re}($ on axis, $x / d=100$ ). effect is not due to resolution difficulties, as will be addressed below.

Another manifestation of a change with $\mathrm{Re}$ is in the corresponding scalar power spectra (Fig. 3). Here, we plot. the spectra $E_{c}\left(f \tau_{\delta}\right)$ at the four Reynolds numbers, normalized by the estimated large-scale times,

$$
\tau_{\delta} \equiv \delta / U_{c l},
$$

and the local mean concentration squared, $\bar{c}^{2}$. The frequency axis has also been nondimensionalized by $\tau_{\delta}$. With these normalizations, equal (equivalent) length scales line up on the $f \tau_{\delta}$ axis, regardless of Re. The large-scale passage frequencies, as independently estimated by the local centerline velocities divided by the width of the jet, are seen to be aligned at $f \tau_{\delta}=1$, corresponding to the length scale $\delta$. So, alternatively, the $f \tau_{\delta}$ axis can be viewed as a nondimensional, inverse length scale, which is the same for all $R e$. This view will be useful in the discussion below.

An important observation is that these spectra do not collapse when normalized in this fashion. The central (midfrequency) portion of the spectra can be seen to bulge upward with decreasing Reynolds number. This might, perhaps, be understandable given the low Re involved and the documented reluctance of the jet spectrum to develop a constant slope region until at much higher Re. However, in addition to the shape differences, there is a discrepancy between the low-frequency levels of the spectra. If the normalization was the cause of these discrepancies, the only "free" parameter would be $\tau_{\delta}$, the estimate of the large-scale time.

Two considerations help to rule out attributing the variations to the value of $\tau_{\delta}$. One is that, since $\tau_{\delta}$ multiplies the frequency but divides the spectrum, changes in $\tau_{\delta}$ shift the normalized spectra along lines of slope -1 . This does not improve the collapse. The second point is that the ratio of the constant level region at the lowest $R e$ to that at the highest $R e$ is greater than 2 . A variation in $\tau_{\delta}$ by this amount is not admissible by the uncertainties in this quantity. Therefore, possible uncertainties in normalization cannot explain the variation in the spectra observed.

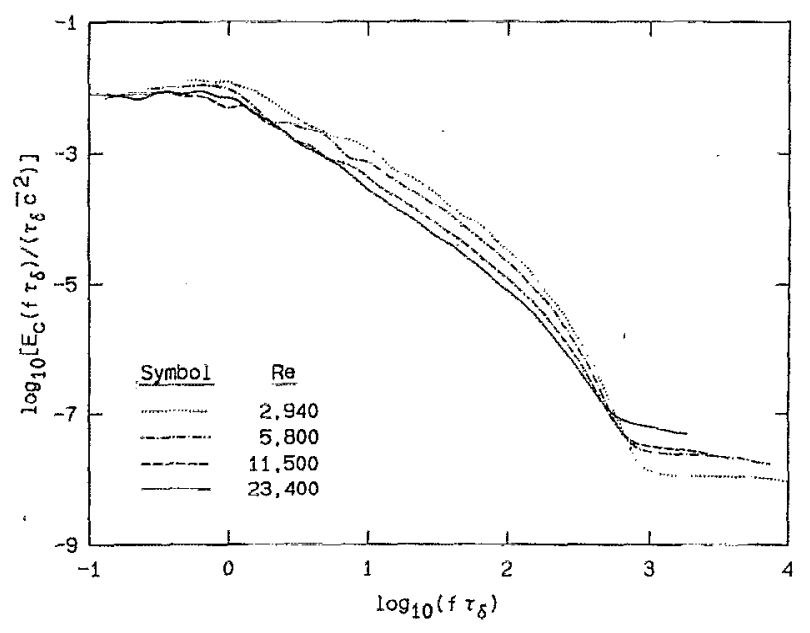

FIG. 3. Normalized scalar power spectra for varying $\operatorname{Re}$ (on axis, $x / d=100$ ). 
Since the area under the power spectrum is equal to $c^{\overline{2}} / \bar{c}^{2}$, the bulging spectra at the lower Re also indicate that $c^{\prime} / \bar{c}$ decreases with increasing Re. It is significant that the area of greatest contribution to this effect is at scales unaffected by potential resolution difficulties, namely, from the variation at the lower frequencies, corresponding to time scales of the order of the large-scale passage time, or length scales close to the local jet diameter.

We calculate the normalized scalar $\mathrm{rms} c^{\prime} / \bar{c}$ from the data and plot it as a function of Re (Fig. 4). These values are, again, from data collected at $x / d=100$, with the exception of one point (fifth from the right), which is from data recorded at $x / d=300$. The resulting $c^{\prime} / \bar{c}$ can be seen to continuously decrease with increasing Re. This is consistent with the behavior of the pdf's and the spectra. The smooth line in Fig. 4 was generated by fitting the measured values of $c^{\prime} / \bar{c}$ using a function of the form

$$
c^{\prime} / \bar{c}=A+B \operatorname{Re}^{p} \text {. }
$$

The values of the fit parameters obtained were $A=0.20$, $B=550$, and $p=-1.02$. The quality of the fit is insensitive to small changes (of several percent) in these parameter values, as measured by the mean squared error's response.

We note that the nonzero value of $A$ indicates that $c^{\prime} / \bar{c}$ may be approaching an asymptotic value of about 0.20 at very large Reynolds numbers. We will return to this point below.

\section{ISSUES OF RESOLUTION}

The issue of resolution for these measurements is crucial and was discussed in Miller and Dimotakis. ${ }^{17}$ To reiterate, the Kolmogorov ${ }^{21}$ scale can be estimated directly from its definition in terms of the mean energy dissipation rate, i.e.,

$$
\lambda_{\mathrm{K}} \equiv\left(v^{3} / \varepsilon\right)^{1 / 4} \text {. }
$$

Using the result of Friehe et al. ${ }^{22}$ for the kinetic energy dissipation rate $\varepsilon$ on the centerline of a turbulent jet, ${ }^{11}$ i.e.,

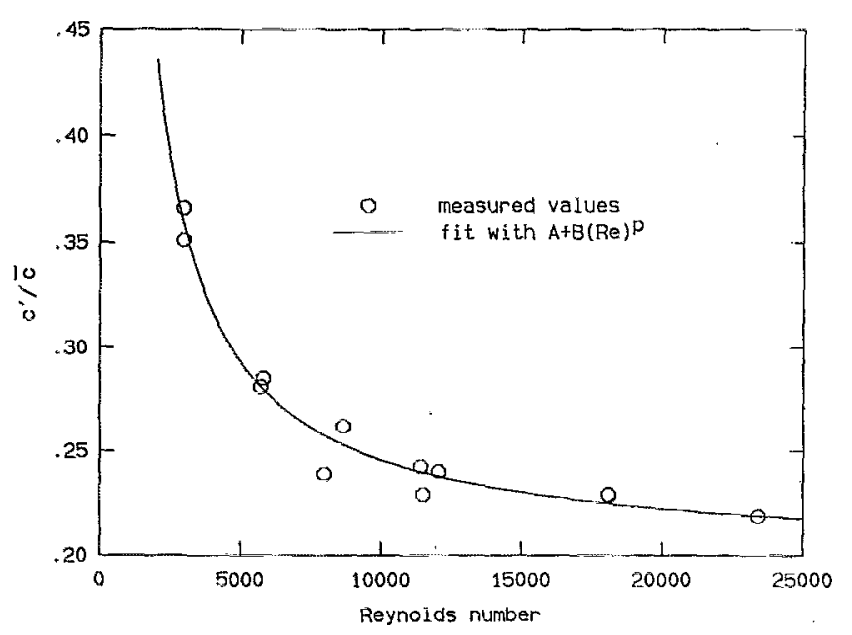

FIG. 4. Normalized scalar rms fluctuations for varying $\operatorname{Re}$ (on axis, $x / d=100$ and 300 ). The data were fit with a function of the form $A+B \operatorname{Re}^{P}$. The parameter values determined by the fit are $A=0.20$, $B=550$, and $p=-1.02$.

$$
\varepsilon=48\left(U_{0}^{3} / d\right)\left[\left(x-x_{0}\right) / d\right]^{-4},
$$

the Kolmogorov scales for these measurements are found to range from roughly 50 to $250 \mu \mathrm{m}$. By similarity arguments, the velocity field spatial scale where the action of viscosity will become important, say, $\lambda_{v}$, will be some multiple of $\lambda_{\mathrm{K}}$. Normalized energy spectra are found to break from a constant power law at a wave number $k_{v}$ such that $k_{v} \lambda_{\mathrm{K}} \approx 1 / 8$ (e.g., Chapman ${ }^{23}$ ). This yields an estimate of

$$
\lambda_{v}=\pi / k_{v} \sim 25 \lambda_{\mathrm{K}} \text {. }
$$

The smallest expected scalar diffusion scale $\lambda$, is smaller yet by a factor of $\mathrm{Sc}^{1 / 2}$ (Batchelor ${ }^{24}$ ), or $\sim 30$ in this case, yielding an estimate for $\lambda_{0}$, very close to $\lambda_{\mathrm{K}}$ (in water). These estimates are corroborated by the gas phase experiments of Dowling and Dimotakis ${ }^{11}$ as well as the measurements in water by Buch and Dahm ${ }^{25}$ and suggest that the smallest diffusion scales may have been resolved in these experiments, at least at the lower Reynolds numbers.

There are three resolution requirements on these measurements: spatial, temporal, and signal-to-noise (SNR). If we convert our spatial resolution estimate to the units of Fig. 3 , using the mean centerline velocities, we obtain values of about $f \tau_{\delta}=1350$. Note that this value is the same on the nondimensionalized frequency axis for all four Re, just as with the large-scale passage times. If we accept that the highest resolvable frequency is half this valuc, we cstimate our spectra are spatially resolved up to about 2.8 on the log scale of Fig. 3. Thus, at the lower Reynolds numbers, the spatial resolution is at least as restrictive as SNR resolution, while for the highest Re, the SNR may be slightly more important in determining the limit of resolution.

To estimate the impact of resolution on these measurements, we utilize a common classical model spectrum. At sufficiently high $\mathrm{Re}$, one might expect the spectrum to have assumed a similarity form. We approximate this as a constant level from zero frequency to the large-scale passage frequency, a region of constant $-5 / 3$ slope, and zero beyond a cutoff at $f_{c} \tau_{\delta}$ (Fig. 5). The constant level value is defined as

$$
K^{2} \equiv E_{c}\left(f \tau_{\delta}<f_{\delta} \tau_{\delta}\right) / \bar{c}^{2} \tau_{\delta}
$$

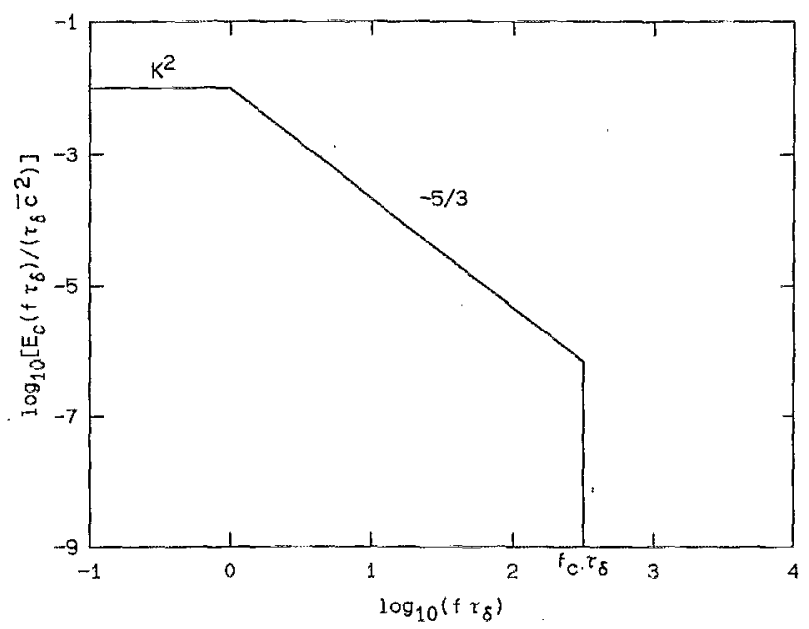

FIG. 5. Model spectrum. 
The spectrum is normalized here to the variance of the scalar fluctuations, i.c.,

$$
\overline{c^{\prime 2}}=2 \int_{0}^{\infty} \frac{E_{c}\left(f \tau_{\delta}\right)}{\tau_{\delta}} d\left(f \tau_{\delta}\right),
$$

which relates the spectrum directly to $c^{\prime} / \bar{c}$. We integrate the model spectrum

$$
\begin{aligned}
\frac{1}{2} \frac{\overline{c^{\prime 2}}}{\overline{c^{2}}}= & \int_{0}^{f_{\delta} \tau_{\delta}} K^{2} d\left(f \tau_{\delta}\right) \\
& +\int_{f_{\delta} \tau_{\delta}}^{f_{\varepsilon} \tau_{\delta}} K^{2}\left(f \tau_{\delta}\right)^{-5 / 3} d\left(f \tau_{\delta}\right)
\end{aligned}
$$

to obtain the result

$$
c^{\prime} / \bar{c}=K\left\{2+3\left[1-\left(f_{\delta} / f_{c}\right)^{2 / 3}\right]\right\}^{1 / 2} .
$$

On the jet axis and at fixed $x / d$, this can be expressed as a function of Reynolds number by substituting for $f_{\delta}$ and $f_{c}$ (cf. Dowling ${ }^{18}$ ), yielding

$$
c^{\prime} / \bar{c}=K\left[2+3\left(1-0.928 \operatorname{Re}^{-1 / 2}\right)\right]^{1 / 2} .
$$

Several things are apparent from Eq. (10). First, it indicates that $c^{\prime} / \bar{c}$ would be expected to increase slightly with Reynolds number. This is opposite to the trend exhibited by our data. Also, we note that at infinite Reynolds number Eq. (10) predicts a finite value for $c^{\prime} / \bar{c}$ of $V 5 K$. To evaluate this expression for our measurements, we fit the level, low-frequency, portion of our highest Re spectrum to determine $K^{2}$, yielding $K^{2} \approx 0.0078$ and an asymptotic value of $c^{\prime} / \bar{c} \approx 0.198$. This value of $K^{2}$ agrees well with the corresponding estimate from gas phase data, ${ }^{11}$ while the asymptotic value of $c^{\prime} / \bar{c}$ agrees with the suggested infinite Re value of 0.20 obtained by fitting the data [parameter $A$ in Eq. (2)]. Finally, the Reynolds number dependence of $c^{\prime} / \bar{c}$ in Eq. (10) is extremely weak. For Re in excess of $10^{3}, c^{\prime} / \bar{c}$ has attained over $99 \%$ of the infinite Re asymptotic estimate.

The model spectrum also helps demonstrate the effect resolution limitations would have on the resulting measure of $c^{\prime} / \bar{c}$. The picture is much the same as in Fig. 5, but we assume that the measured spectrum is resolution-limited at some nondimensional frequency $f_{r} \tau_{\delta}$ (Fig. 6). The lost contribution to $c^{\prime} / \bar{c}$, represented by the area under the spectrum between $f_{r} \tau_{\delta}$ and $f_{c} \tau_{\delta}$, i.e.,

$$
\Delta c^{\prime} / \bar{c}=c^{\prime} / \bar{c}-\left[c^{\prime} / \bar{c}\right]_{r},
$$

is equal to

$$
\Delta c^{\prime} / \bar{c}=\sqrt{3} K\left[\left(f_{\delta} / f_{r}\right)^{2 / 3}-\left(f_{\delta} / f_{c}\right)^{2 / 3}\right]^{1 / 2},
$$

while the ratio of the resolution-limited value and the true value is given by

$$
\frac{\left[c^{\prime} / \bar{c}\right]_{r}}{c^{\prime} / \bar{c}}=\left(\frac{2+3\left[1-\left(f_{\delta} / f_{r}\right)^{2 / 3}\right]}{2+3\left[1-\left(f_{\delta} / f_{c}\right)^{2 / 3}\right]}\right)^{1 / 2} .
$$

Here, again, the ratio $f_{\delta} / f_{c}$ is very small for the Reynolds numbers of interest. As a result, if the ratio $f_{\delta} / f_{r}$ is also kept small; resolution can hardly affect the measured $c^{\prime} / \bar{c}$ at these $\mathrm{Re}$, because the expression in Eq. (13) is then very close to unity. Also note that if the scalar spectrum is adequately described by the model spectrum and a particular Reynolds number measurement is resolution limited, higher

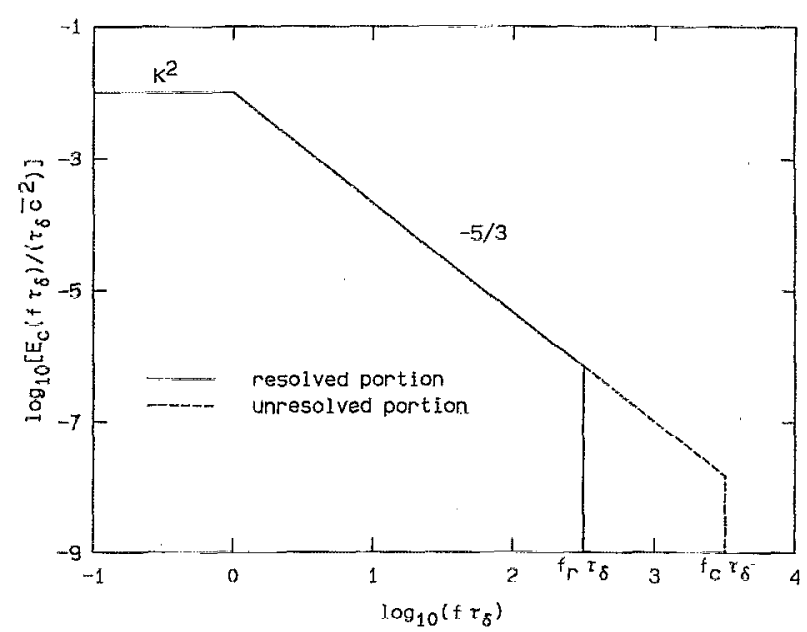

FIG, 6. Resolution-limited model spectrum.

Re data should yield the same (measured) value of $\left[c^{\prime} / \bar{c}\right]_{\text {r }}$ (not less). This is clear because the measured spectra should then have the same shape and same extent, correspondingly yielding the same values of $\left[c^{\prime} / \bar{c}\right]_{r}$. If a Batchelor $k^{-1}$ region $^{24}$ was incorporated into the model spectrum, it would not alter these results at the present Schmidt number of about $10^{3}$ (see Moffat ${ }^{26}$ ). We conclude that our measurements of $c^{\prime} / \bar{c}$ are not significantly affected by resolution difficulties.

Finally, recall that we have argued that we may, in fact, be resolving the smallest diffusion scales in the flow, at least for the lower Reynolds number flows we have investigated. Specifically, we note that our measured spectra do not appear to support the $k^{-1}$ Batchelor ${ }^{24}$ prediction at high wavc numbers (frequencies). This observation is significant at the value of the Schmidt number applicable here, since $\mathrm{Sc}^{1 / 2} \sim 30$. This would have yielded a $k^{-1}$ portion in the spectrum spanning roughly 1.5 decades in frequency. If our assessment of the resolution of our measurements, relative to the requisite physical scales, is correct, then the resolution and SNR of our measurements, at least at the lower Reynolds numbers, are sufficient to have elucidated this behavior. As regards the transport of scalars characterized by low diffusivity (large $\mathrm{Sc}$ ) in turbulent jets in the Reynolds number range we have investigated, we are forced to conclude that they do not display a $k^{-1}$ Batchelor spectrum.

This finding should be examined in the context of a theoretical difficulty with the $k^{-1}$ spectrum. In particular, the Batchelor spectrum does not appear to exhibit the correct limiting behavior at high Schmidt numbers (holding all other flow parameters fixed). As Batchelor had noted, ${ }^{24}$ the variancc computed using the $k^{-1}$ spectrum diverges logarithmically with increasing Sc. This divergence, however, is inconsistent with the required behavior of scalar fluctuations, which must remain bounded, irrespective of the value of the Schmidt number, as noted recently. Briefly, ${ }^{27}$ the variance $\overline{c^{2}}$ of a scalar bounded by $c=0$ and $c=1$ by the initial/boundary conditions is bounded, in turn, by

$$
\max \left\{c^{\overline{2}}\right\}=\bar{c}(1-\bar{c}) \text {. }
$$


This maximum is expected in the limit of (identically) zero diffusivity, $\mathscr{D} \rightarrow 0$, or $S c \rightarrow \infty$ if all other flow parameters are held fixed, and corresponds to a limiting pdf of

$$
p(c) \rightarrow(1-\bar{c}) \delta(c)+\bar{c} \delta(1-\bar{c}),
$$

i.e., stirring but no (molecular) mixing by the turbulent flow. A logarithmic divergence of $\overline{c^{\prime 2}}$ as $\mathrm{Sc} \rightarrow \infty$, as estimated by the integral over the $k^{-1}$ scalar spectrum, is inconsistent with the bound of Eq. (14).

\section{CONCLUSIONS}

We have conducted high-resolution, single-point, scalar measurements on the centerline in the far field of an axisymmetric, turbulent jet at high Schmidt number $\mathrm{Sc} \sim 10^{3}$, over a range of Reynolds numbers between 3000 and 24000 . The jet (Taylor) microscale Reynolds number, estimated by ${ }^{11}$

$$
\mathrm{Re}_{\mathrm{T}} \approx 1.4 \mathrm{Re}^{\mathrm{I} / 2}
$$

was, therefore, approximately 80 at $\operatorname{Re}=3000$ and 220 at $\mathrm{Re}=24000$. Except, perhaps, for the lowest values, such microscale Reynolds numbers are regarded as high by some turbulence criteria. ${ }^{28}$ Nevertheless, our experiments indicate that, in a number of ways, at least at high Schmidt numbers, jet mixing has not attained asymptotic behavior by $\operatorname{Re} \approx 2.4 \times 10^{4}$. In particular, pdf's and spectra of $c / \bar{c}$, the normalized jet fluid concentration, are found to depend on Rcynolds number. The rms concentration fluctuations $c^{\prime} / \vec{c}$ were found to continuously decrease with increasing $R e$, over the Reynolds number range examined.

We have argued, on the basis of our spectral measurements and the use of a simple model spectrum, that these results are not an artifact of inadequate resolution. In particular, the Reynolds number dependence of $c^{\prime} / \bar{c}$, as estimated on the basis of the model spectrum, should have exhibited the opposite behavior to that observed. The limiting values of $c^{\prime} / \bar{c}$ at high $\mathrm{Re}$, from both the model and the experimental estimates, agreed quite well, suggesting that $c^{\prime} / \bar{c} \approx 0.20$ may represent an asymptotic value for the normalized rms of the jet fluid concentration fluctuations, at $S c \sim 10^{3}$, on the jet axis. Our measurements did not extend to jet Reynolds numbers high enough to confirm such asymptotic behavior directly.

As noted earlier, measurements in gas phase jets at comparable Reynolds numbers ${ }^{11}$ suggest that, at least for $\mathrm{Sc} \sim 1$, the pdf of jet fluid concentration does not exhibit as strong a Reynolds number dependence as we are reporting here, if any at all. This difference in behavior may, of course, be a manifestation of Schmidt number effects. At this time, we cannot reconcile the disagreement between the Reynolds number dependence of the present results and the liquid phase "flame length" data of Weddell ${ }^{8}$ (see also Hottel, Ref. 9), and Dahm et al. ${ }^{29}$ In particular, on the basis of our measured pdf 's of concentration, we would conclude that, in liquid phase $\left(\mathrm{Sc} \sim 10^{3}\right)$ jets, one should observe decreasing flame lengths with increasing Reynolds number, at least within the Reynolds number range investigated here (see Appendix B). We note, however, that Kristmanson and Danckwerts ${ }^{10}$ found the flame length to vary with Re until about twice as large a Reynolds number as Weddell, and Dahm et al.

In summary, we have found that the pdf 's of the jet fluid concentration become narrower with increasing Reynolds number. A measure of this trend is provided by $c^{\prime} / \bar{c}$, the normalized rms level of scalar fluctuations, which decreases as the Reynolds number increases. These measures of unmixedness suggest a more homogeneous turbulent, liquid phase, jet fluid concentration field, i.e., better molecular mixing, with increasing Reynolds number. Our spectra also evolve with increasing Reynolds number, developing a constant slope, close to $-5 / 3$, at the higher values.

Finally, we did not observe a $k^{-1}$ spectral behavior at the small scales, ${ }^{24}$ even though the predicted range of validity of this regime would be quite large at this Schmidt number and falls within the resolution range of our measurements at the lower Reynolds numbers that were investigated.

\section{ACKNOWLEDGMENTS}

We would like to acknowledge the contributions of D. Lang to the experiments, as well as helpful discussions with J. E. Broadwell and A. R. Kerstein.

This work was performed under AFOSR Grants No. 83-0213 and No. 88-0155, and GRI Contract No. 5087-2601467.

\section{APPENDIX A: ISSUES OF BUOYANCY}

An experimental consideration that was addressed, in addition to resolution, is buoyancy. There are two cases to be considered: buoyancy from a jet fluid/reservoir fluid density difference, and possible heating of the jet at the measuring station by the laser beam. Briefly, a nondimensionalized buoyancy length scale, ${ }^{30}$

$$
\frac{L_{b}}{d} \equiv\left(\frac{\pi \rho_{j}}{4 \rho_{\infty}}\right)^{1 / 4}\left(\frac{\rho_{j} U_{0}^{2}}{\left(\rho_{j}-\rho_{\infty}\right) g d}\right)^{1 / 2},
$$

where $\rho_{j}$ the jet fluid density, $\rho_{\infty}$ the reservoir fluid density, $U_{0}$ is the jet exit velocity, and $g$ the acceleration of gravity, may be derived from dimensional analysis (e.g., Dowling, ${ }^{18}$ Sec. A.5). This parameter may then be compared with experimentally derived values to determine the role of buoyancy in the flow. In particular, Papanicolaou and List ${ }^{14,15}$ find that jets are momentum-dominated if $x / L_{b}$ is less than one, while Chen and Rodi ${ }^{31}$ cite a limit of roughly 0.5 .

We will estimate the maximum anticipated density difference and calculate the resulting value of $L_{b} / d$. The density difference may arise from the addition of the heavy dye to the jet fluid, or from a temperature mismatch between the jet fluid and the reservoir fluid. Let us consider the two contributions separately, since, it turns out, they are of different magnitudes. The fluorescein dye concentration used in the cxperiments was roughly $3.3 \times 10^{-7} \mathrm{M}$. The formula weight of disodium fluorescein is 376.28 . This yields an estimate for the dye-induced buoyancy length scale [from Eq. (A1)] of over 19600 jet nozzle diameters at the lowest Re. For each run, the jet fluid was prepared by diluting a small amount of 
concentrated stock solution of dye with sufficient water, taken from the reservoir shortly before the run, to yield the proper jet dye concentration. This procedure resulted in no observable temperature discrepancy between the plenum fluid and the reservoir fluid. Using Eq. (A1), the estimated buoyancy length scale resulting from a temperature difference of $0.1^{\circ} \mathrm{C}$, the accuracy of the thermometer, is 1500 jet nozzle diameters. While, of the two contributions, the possible influence of a worst-case temperature mismatch is larger, the resulting buoyancy length is 15 times the distance between the nozzle and the measurement location at $x / d=100$. Therefore, based on the criterion of Chen and Rodi, ${ }^{31}$ the momentum-dominated region extends for a distance that is over seven times longer.

A second way buoyancy may arise is through heating of the fluid in the measuring volume via absorption of laser power by the dye. The approximate residence time of the fluid in the measurement volume is the measurement height $(80 \mu \mathrm{m})$ divided by the calculated mean centerline velocity at the lowest Reynolds number $(0.072 \mathrm{~m} / \mathrm{sec})$, or $0.001 \mathrm{sec}$. We estimate the laser attenuation as no more than $5 \%$ across the width of the jet, and treat the jet concentration as uniform in the radial direction for the purposes here (not too severe an approximation-cf. $\left.\mathrm{Dahm}^{12}\right)$. The attenuation across the measuring volume $\left(5 \times 10^{-7} \mathrm{~cm}^{3}\right)$ would then be about $0.005 \%$. Assuming a worst case (all power attenuation goes into heat), the resulting temperature rise would then be approximately $0.02^{\circ} \mathrm{C}$. While the approximation of uniform concentration and velocity may tend to underestimate the temperature rise, the actual laser attenuation through the entire jet diameter is closer to $3 \%$, or so, with less than half of the attenuated power converted into heat. Therefore this estimate is conservative.

A second point concerning the heating of the fluid by the laser beam is that, while some dilatation of the fluid occurs as it passes through the laser beam, it is a localized effect. Importantly, there is no global buoyancy force to affect the large-scale jet structure and entrainment, as there is when the jet and reservoir densities are different. Thus it is not appropriate to utilize criteria such as those of Chen and Rodi $^{31}$ in this case, since they apply to the situation with globally different densities. The primary influence here, i.e., local dilatation, will have an even weaker effect on the flow for a given temperature rise. The dilatation is extremely small, $\Delta \rho / \rho \approx 5 \times 10^{-6}$, and we argue that its influence in our flow environment is also insignificant.

Having addressed the issue of attenuation, we should also consider the possibility of attenuation-caused fluctuations of the laser beam contributing to the measured values of $c^{\prime} / \bar{c}$. The beam attenuation across the diameter of the jet was typically $3 \%$, or less. Fluctuations arising from this attenuation may be completely uncorrelated with the scalar fluctuations at the measurement location, in which case the variances of the two contributions add. Alternatively, the fluctuations may be exactly correlated, in which case the two contributions to $c^{\prime} / \bar{c}$ add. In either situation, the maximum influence would correspond to fluctuations between the minimum (zero) and maximum $(\sim 1.5 \%)$ attenuation possibilities, or a maximum correction to the value of $c^{\prime} / \bar{c}$ of roughly 0.015 . This would result in, at most, a $7 \%$ change in the measured value. This should be compared with the large percentage decrease in the measured values over the Reynolds number range investigated (Fig. 4). Therefore, even in a worst-case scenario, the fluctuations arising from the attenuation of the laser beam would represent only a small correction to the magnitude of the variation we have measured. The actual situation is obviously not nearly so adverse, and we conclude that the attendant contamination of our results, as a result of beam fluctuations, is extremely small.

\section{APPENDIX B: INTERPRETATION OF FLAME LENGTHS}

Given the jet fluid concentration pdf's $p(c, x ; \operatorname{Re})$, the "flame length" can be estimated as the distance required for $1-\epsilon$ of the jet fluid (with, say, $\epsilon=0.01$ ) to have mixed to the proper composition (jet fluid fraction) $c_{r}$, as required by the chemical reaction. This is related to the stoichiometric mixture ratio (equivalence ratio) $\phi_{r}$ by the expression

$$
c_{r}=\phi_{r} /\left(\phi_{r}+1\right) \text {, }
$$

where $\phi_{r}$ is equal to the parts of reservoir fluid that must be molecularly mixed and reacted for the complete consumption of one part of (pure) jet fluid.

Assuming that the highest values of the jet fluid concentrataion occur on the jet axis, to estimate the flame length corresponding to a given $c_{r}$ and $\epsilon$, we need two additional assumptions. First, that the pdf of concentration on the jet axis is a function of $c / \bar{c}$ only, i.e,

$$
p(c, x) \approx f(c / \bar{c}),
$$

where the function of $f(c / \bar{c})$ may vary with Reynolds number (and Schmidt number), but is taken as independent of $x / d$. Note that it is $f(c / \bar{c})$ that was plotted under this implicit assumption in Fig. 2. This is the usual assumption, recently corroborated for gas phase $(\mathbf{S c} \sim 1)$ turbulent jets, ${ }^{11}$ with supporting evidence for liquid phase $\left(\mathrm{Sc} \sim 10^{3}\right)$ jets. ${ }^{14}$ The second assumption deals with the dependence of $\bar{c}$ on $x / d$, i.e. (momentum-driven turbulent jets)

$$
\bar{c}=\kappa d^{*} /\left(x-x_{0}\right) .
$$

In this expression, the (pure) jet fluid concentration is taken as unity, the dimensionless parameter $\kappa$ is taken as a constant (which may, however, depend on Reynolds number), ${ }^{11} d^{*}$ is the jet momentum diameter, ${ }^{6,7,12}$ and $x_{0}$ is a virtual origin (possibly weakly dependent on Reynolds number at low Reynolds numbers). Combining Eqs. (B2) and (B3), we have, for each Reynolds number

$$
p(c, x) \approx f\left\{\left[\left(x-x_{0}\right) / k d^{*}\right] c\right\} .
$$

For a given $\epsilon$, the flame length $L_{f}$ may then be estimated as the implicit solution of the equation

$$
\int_{c_{r}}^{\infty} p\left(c, L_{f}\right) d c=\epsilon .
$$

It can be seen that the computed flame length is determined by the behavior of the pdf at high values of $c / \bar{c}$. Basically, if $f_{i}(c / \bar{c})$ is the pdf of $c / \bar{c}$ at a Reynolds number $\operatorname{Re}_{j}$, then for $c_{r} / \bar{c}>1$ (small $\epsilon$ ) and $f_{2}\left(c_{r} / \bar{c}\right)>f_{1}\left(c_{r} / \bar{c}\right)$, we will have $L_{f 2}>L_{f 1}$. In other words, and as perhaps obvious in retro- 
spect, a larger $c^{\prime} / \bar{c}$ (less well-mixed jet) implies a longer flame length.

The inferred flame length dependence on Reynolds number made above assumes that any deviations of the $x$ dependence of $p(c, x)$ from Eq. (B2), as well as the possible dependence of $d^{*}, \kappa$, and $x_{0}$ on Reynolds number, are not strong enough to offset the relatively large dependence of $L_{f}$ predicted using Eq. (B4) and the assumptions outlined above. We note, however, that the validity of these assumptions may not be unassailable, especially at the high Schmidt number in this experiment. ${ }^{27}$

'H. L. Toor, AIChE J. 8, 70 (1962).

${ }^{2}$ J. E. Broadwell and M. G. Mungal, Phys. Fluids A 3, 1193 (1991).

${ }^{3}$ M. G. Mungal and P. E. Dimotakis, J. Fluid Mech. 148, 349 (1984).

${ }^{4}$ M. M. Koochesfahani and P. E. Dimotakis, J. Fluid Mech. 170, 83 (1986).

${ }^{5}$ M. G. Mungal, J. C. Hermanson, and P. E. Dimotakis, AIAA J. 23, 1418 (1985).

${ }^{\circ}$ W. J. A. Dahm and P. E. Dimotakis, AIAA J. 25, 1216 (1987),

${ }^{7}$ W. J. A. Dahm and P. E. Dimotakis, AIAA Paper No. 85-0056, 1985.

${ }^{8} \mathrm{D}$. Weddell, Ph.D. thesis, Massachusetts Institute of Technology, 1941.

${ }^{9} \mathrm{H}$. C. Hottel, The 4th (International) Sympositum on Combustion (Williams and Wilkins, Baltimore, 1953), pp. 97-113.

${ }^{10}$ D. Kristmanson, and P. V. Dankwerts, Chem. Eng. Sci. 16, 267 ( 1961 ).

"D. R. Dowling and P. E. Dimotakis, J. Fluid Mech. 218, 109 (1990).

${ }^{12}$ W. J. A. Dahm, Ph.D. thesis, California Institute of Technology, 1985.
${ }^{13}$ F. C. Lockwood and H. A. Moneib, Combust. Sci. Technol. 22, 63 (1980).

${ }^{14}$ P. N. Papanicolaou and E. J. List, Int. J. Heat Mass Transfer 30, 2059 (1987).

${ }^{15}$ P. N. Papanicolaou and E. J. List, J. Fluid Mech. 195, 341 (1988).

${ }^{16}$ D. A. Papantoniou, Ph.D. thesis, California Institute of Technology, 1985.

${ }^{17}$ P. L. Miller and P. E. Dimotakis, Phys. Fluids A 3, 168 (1991).

${ }^{18}$ D. R. Dowling, Ph.D. thesis, California Institute of Technology, 1988.

${ }^{19}$ N. Wiener, Extrapolation. Interpolation and Smoothing of Stationary Time Series (Wiley, New York, 1949).

${ }^{20}$ W. H. Press, B. P. Flannery, A. A. Teukolsky, and W. T. Vetterling, $\mathrm{Nu-}$ merical Recipes. The Art of Scientific Computing (Cambridge U. P., New York, 1986).

${ }^{21}$ A. N. Kolmogorov, Dokl. Akad. Nauk SSSR 30, 299 (1941); [Usp. Fiz. Nauk 93, 476 (1967)] [Sov. Phys. Usp. 10, 734 (1968)].

${ }^{22}$ C. A. Friehe, C. W. Van Atta, and C. H. Gibson, AGARD Turbulent Shear Flows CP-93, 18.1-7, 1971.

${ }^{23}$ D. R. Chapman, AlAA J. 17, 1293 (1979).

${ }^{24}$ G. K. Batchelor, J. Fluid Mech. 5, 113 (1959).

${ }^{25}$ K. A. Buch and W. J. A. Dahm, Bull. Am. Phys. Soc. 34, 2264 (1989).

${ }^{26}$ H. K. Moffatt, Rep. Prog. Phys. 46, 621 (1983).

${ }^{27}$ P. E. Dimotakis and P. L. Miller, Phys. Fluids A 2, 1919 (1990).

${ }^{28}$ K. R. Sreenivasan, Phys. Fluids 27, 1048 (1984).

${ }^{99}$ W. J. A. Dahm, P. E. Dimotakis, and J. E. Broadwell, AIAA Paper No. 84-0369, 1984.

${ }^{30}$ H. B. Fischer, E. J. List, R. C. Y. Koh, J. Imberger, and N. H. Brooks, Mixing in Inland and Coastal Waters (Academic, New York, 1979).

${ }^{31}$ C. J. Chen and W. Rodi, Vertical Turbulent Buoyant Jets. A Review of Experimental Data (Pergamon, Oxford, 1980). 